\title{
EFEITO DE Bixa orellana NA ALTERAÇÃO DE CARACTERÍSTICAS DE OVOS DE GÁLINHAS
}

\author{
Effect of Bixa orellana in the alteration of characteristics of poultry laying eggs
}

\author{
Marcia Nalesso Costa Harder ${ }^{1}$, Solange Guidolin Canniatti Brazaca ${ }^{2}$, \\ Vicente José Maria Savino ${ }^{3}$, Antonio Augusto Domingos Coelho ${ }^{4}$
}

\begin{abstract}
RESUMO
Objetivou-se, no presente trabalho, avaliar os efeitos da adição de urucum (Bixa orellana L.) na ração de galinhas poedeiras, quanto à possível interferência na qualidade dos ovos. Para obtenção das amostras foram utilizados 125 animais, divididos em cinco tratamentos: Controle (0\% - T1) e quatro tratamentos com adição de urucum na ração $(0,5 \%$ - T2; 1,0\% - T3; 1,5\% - T4 e 2,0\% - T5). Os animais foram separados aleatoriamente em cinco blocos de cinco animais, totalizando 25 animais por parcela. Os ovos, após coletados, foram classificados usando ovoscópio, pesados, realizada análise de gravidade específica, unidade Haugh, altura de albúmen e gema, espessura da casca, diâmetro e índice de gema. As diferentes dietas não afetaram: peso; gravidade específica; altura do albumén; espessura da casca; altura de gema; diâmetro de gema. Pôde ser observado que a adição de urucum, na ração de poedeiras, não alterou a maior parte dos parâmetros de qualidade física do ovo, somente a unidade Haugh e o índice de gema apresentaram diferença $(\mathrm{p} \leq 0,05)$
\end{abstract}

Termos para indexação: Urucum, poedeiras, qualidade de ovos.

\section{ABSTRACT}

This research evaluated the effects of addition of anatto (Bixa orellana L.) to feed laying hens to verify the possible interference of the anatto on egg quality. The eggs were obtained from 125 animal divided in 5 treatments: Control (0\% - T1) and four treatments with addition of anatto $(0.5 \%$ - T2; $1.0 \%$ - T3; $1.5 \%$ - T4 and 2.0\% - T5). The laying hens were separated in five blocks, each block with 5 animals, with total 25 animals by parcel. The eggs picked were classified using eggscopic, weighed, specific gravity, Haugh unit, albumen and yolk height, thickness of the shell, diameter and index yolk. Different feeds did not modify: weight; specific gravity; albumen height, thickness of the shell; yolk height and diameter of yolk. About the eggs quality analysis, addition of anatto did not show significance ( $p>0.05$ ) among the treatments to most of the parameters of physical quality of eggs, only Haugh unit and yolk index showed differences $(\mathrm{p} \leq 0.05)$.

Index terms: Anatto, laying hens, eggs quality.

(Recebido em 9 de março de 2006 e aprovado em 26 de fevereiro de 2008)

\section{INTRODUÇÃO}

Os consumidores dão preferência aos ovos com gema amarelo-alaranjadas e frangos com pele bem pigmentada. Os pigmentos são freqüentemente agregados às rações das aves quando o sorgo substitui o milho, total ou parcialmente, pela preferência do mercado, embora não representando valor nutritivo (ENGLERT, 1998).

Segundo Joly (1993), o urucum (Bixa orellana L.) é o único gênero pertencente à família Bixaceae, nativo da América Tropical e extremamente cultivado. Da produção brasileira, cerca de $70 \%$ dos grãos produzidos destinam- se ao processamento do colorau (corante alimentar de uso doméstico), 20\% são utilizados na produção do corante e $10 \%$ são exportados. Dessa forma, a produção in natura é muito pequena, não conseguindo, às vezes, suprir o mercado interno (BATISTA, 1994). Há necessidade de se aumentar a produção, além do emprego de tecnologias para obter produtos de qualidade. A necessidade tende a aumentar, em razão da tendência atual de substituir os corantes artificiais pelos naturais. $\mathrm{O}$ alto custo dos pigmentos sintéticos usados atualmente, os efeitos prejudiciais que eles causam à saúde humana e animal, a necessidade de maior diversificação agrícola, e a escassa

\footnotetext{
Engenheira Agrônoma, Mestre em Ciência e Tecnologia de Alimentos - Departamento de Alimentos e Nutrição Humana/LAN - Escola Superior de Agricultura "Luiz de Queiroz"/ESALQ - Universidade de São Paulo/USP - Avenida Pádua Dias, 11 - Agronomia - Cx. P. 09 - 13418-900 - Piracicaba, SP_marciancosta@ig.com.br

${ }^{2}$ Nutricionista, Doutora em Ciência dos Alimentos, Professora - Departamento de Alimentos e Nutrição Humana/LAN - Escola Superior de Agricultura "Luiz de Queiroz"/ESALQ - Universidade de São Paulo/USP - Avenida Pádua Dias, 11 - Agronomia - Cx. P. 09 - 13418-900 - Piracicaba, SP sgcbraza@esalq.usp.br

${ }^{3}$ Médico Veterinário, Doutor, Professor Associado - Departamento de Genética/LGN - Escola Superior de Agricultura "Luiz de Queiroz"/ESALQ Universidade de São Paulo/USP - Avenida Pádua Dias, 11 - Agronomia - Cx. P. 09 - 13418-900 - Piracicaba, SP - vjmsavin@esalq.usp.br ${ }^{4}$ Engenheiro Agrônomo, Doutor, Professor - Departamento de Genética/LGN - Escola Superior de Agricultura "Luiz de Queiroz"/ESALQ - Universidade de São Paulo/USP - Avenida Pádua Dias, 11 - Agronomia - Cx. P. 09 - 13418-900 - Piracicaba, SP - aadcoelh@esalq.usp.br
} 
informação existente sobre a utilização do urucum, como fonte de cor para a gema do ovo, foram os principais fatores que motivaram Araya et al. (1977) a investigar sobre o assunto.

O fruto contém proteínas, beta-caroteno e outros carotenóides, sendo os mais abundantes a Bixina (cor amarela) e a Norbixina (cor vermelha). Seus principais componentes são: vitamina C $0,05 \%$, proteína $6,61 \%$, açúcares totais $10,24 \%$, ferro $0,08 \%$, óleo essencial $0,05 \%$, resina $1,65 \%$, e tanino em pequena quantidade. Em doses de $500 \mathrm{mg}$ a $1000 \mathrm{mg} / \mathrm{kg}$, por via intraperitoneal em ratos, o urucum (B. orellana) provoca diminuição da atividade motora e aumento da diurese, sem nenhum sinal de toxicidade aparente. A DL (dose letal) no rato, por via intraperitoneal é de 700mg/kg (CATÁLOGO RURAL, 2005).

FAO (1991) e Franco et al. (2002) discutem sobre a legislação para o uso de corantes naturais. Quanto à adoção de corantes e outros aditivos, a legislação brasileira está respaldada nas recomendações do Comitê FAO/OMS "Joint Expert on Food Additives - JECFA". Esse comitê elaborou, ao lado das especificações de identidade e pureza, as condutas a serem observadas nos estudos e avaliações toxicológicas. Para os corantes naturais, essa avaliação deve ser considerada em três grupos:

a) corante isolado quimicamente inalterado de um alimento e usado no produto, em níveis normalmente nele encontrados, não sendo necessários dados toxicológicos;

b) corante isolado quimicamente de um alimento e usado no produto em níveis superiores aos normalmente nele encontrados; deve ser avaliado como se fosse artificial;

c) corante isolado de um alimento, porém, quimicamente modificado durante a sua obtenção ou extraído de outra fonte não alimentar; deve ser avaliado toxicologicamente, como se fosse corante artificial.

De acordo com a legislação vigente, o urucum se enquadra na classificação "a".

Aliando-se à idéia atual de alimentos mais saudáveis e naturais a um alimento nutricionalmente completo, neste trabalho, objetivou-se avaliar os efeitos da adição de urucum (Bixa orellana L.) na ração de galinhas poedeiras, verificando uma possível interferência na qualidade física dos ovos.

\section{MATERIAL E MÉTODOS}

\section{Material}

O trabalho foi realizado na Escola Superior de Agricultura "Luiz de Queiroz" - ESALQ/USP, Piracicaba SP, na área experimental de Sertãozinho, do Departamento de Genética, onde se localiza a granja experimental de aves. Foram utilizadas 125 galinhas poedeiras da raça Hy-Line brown, em idade de início de postura (por volta de 20 semanas), alimentadas diariamente com ração comercial, urucum inteiro (in natura) e amido de milho (como inerte), nas concentrações de 0,5 (T2); 1,0 (T3); 1,5 (T4) e 2,0\% (T5) de urucum em relação ao peso da ração e grupo controle (T1), recebendo somente ração comercial, balanceada adequadamente para atingir o objetivo. Foi fornecida ração para aves de postura em produção, na quantidade de 80 a $120 \mathrm{~g}$ por ave ao dia, seguindo sempre as recomendações de consumo da linhagem. O urucum começou a ser administrado no início da $20^{\mathrm{a}}$ semana. Os animais foram divididos em 5 repetições, com 5 animais cada. Foram alojadas em gaiolas individuais, submetidas a um programa de iluminação artificial e fornecida água à vontade. A ração foi fornecida na totalidade, pela manhã, ao redor das 7 horas. As coletas dos ovos foram feitas logo após o fornecimento da ração até por volta das 12 horas. Os ovos da $28^{\mathrm{a}}$ semana foram utilizados para fazer as análises.

\section{Preparo das amostras}

Com exceção da análise no ovoscópio e da densidade, que exigem a integridade do ovo, para as demais análises, os ovos foram quebrados e utilizados de acordo com cada metodologia.

\section{Peso}

Para as análises de qualidade física, foram coletados 70 ovos de cada tratamento. Foram separados por repetição e pesados em uma balança digital semi-analítica com graduação de $0,01 \mathrm{~g}$. Foi considerado o peso dos ovos inteiros com casca.

\section{Ovoscopia}

Para essa determinação, o ovo foi colocado na frente da abertura da luz, segurando-se o mesmo com o dedo polegar na parte mais fina e com os dedos indicador e médio na parte mais larga. A seguir, foram dadas duas voltas rápidas no ovo a fim de fazer o conteúdo (clara e gema) se mover (OLIVEIRA et al., 2001). Foi utilizado um modelo de ovoscópio caseiro.

\section{Gravidade específica}

Os ovos inteiros, depois de realizada a pesagem e análise em ovoscópio, foram submetidos à análise de gravidade específica. O valor foi obtido pela imersão dos ovos em baldes plásticos, que continham diferentes soluções salinas, com densidades que variam de 1,0650 a 1,0950, com intervalo de 0,0025. As concentrações das 
soluções salinas foram ajustadas periodicamente com a utilização de um decímetro de petróleo para líquidos. Os ovos foram submersos em cestas plásticas nos baldes, da menor para maior concentração salina, e foram retirados ao flutuarem até a superfície, sendo então o valor anotado. Os ovos passaram por balde que continha água pura, que recebe o nome de solução prévia, antes de seguirem para soluções salinas. A gravidade específica foi realizada em ovos frescos, coletados no dia, para que não houvesse interferência de tempo de postura. As soluções salinas foram preparadas conforme recomendação feita por Hamilton (1982).

\section{Altura de albúmen e gema}

Os ovos, após a medida da gravidade específica, foram quebrados em cima de uma placa plana de vidro para mensuração de valores de altura de albúmen e da gema.

O procedimento para a determinação da altura do albúmen consiste em medir na região mediana, entre a borda externa e a gema e, que essa região esteja perpendicular à chalaça (BOARD et al., 1994; SECHINATO, 2003). Foi utilizado um micrometro para medir a altura de albúmen. A altura da gema foi mensurada na região central. O diâmetro da gema foi obtido com paquímetro. Essas medidas foram mensuradas em intervalo de medida de $1 \mathrm{~mm}$. Esses valores foram utilizados, respectivamente, para fins de cálculo da unidade Haugh e índice de gema.

\section{Unidade Haugh}

A Unidade Haugh (UH) relaciona diretamente o peso dos ovos (g) com a altura de albúmem (mm). É utilizado para verificar a qualidade dos ovos, pois, à medida que o ovo se deteriora, a clara se espalha, resultando num menor valor para esse índice. Para a unidade Haugh, o logarítimo de altura da albumina espessa é ajustado cem vezes para ser equivalente àquela de um ovo de 56g (GRISWOLD, 1972). Pode ser utilizada a seguinte equação: $U H=100 \log$ $\left(\mathrm{h}+7,57-1,7 \mathrm{~W}^{0,37}\right)$, onde $\mathrm{h}=$ altura de albúmem $(\mathrm{mm})$ e W = peso do ovo (g). Quanto maior o valor da UH, melhor será a qualidade dos ovos, que são classificados em ovos tipo AA (100 até 72$),$ A (71 até 60), B (59 até 30), C (29 até 0) (SILVA, 2004).

\section{Índice de gema}

O índice de gema (IG) é dado por: IG = altura da gema $(\mathrm{mm})$ / diâmetro da gema $(\mathrm{mm})$. Valores considerados normais vão de 0,3 a 0,5 (SILVA, 2004).

\section{Espessura da casca}

De acordo com a espessura da casca, tem-se um indicativo da fragilidade da mesma; para essa determinação foram utilizadas cascas de ovos quebradas ao meio, incluindo as membranas internas, lavadas em água corrente e secas a temperatura ambiente. Sua leitura foi realizada com micrômetro, em dois pontos na área centro-transversal da casca do ovo, obtendo-se a média, expressa em milímetros $(\mathrm{mm})$

\section{RESULTADOS E DISCUSSÃO}

Os valores dos resultados obtidos são apresentados nas Tabelas 1 e 2 . A maioria das características de qualidade avaliadas não apresentaram diferença $(p>0,05)$ entre os tratamentos, indicando que a adição de urucum na alimentação das poedeiras não interferiu na qualidade dos ovos. As exceções foram a Unidade Haugh e o Índice de gema, que apresentaram diferença significativa entre os tratamentos $(\mathrm{p} \leq 0,05)$, porém aparentemente não ligadas à adição do urucum.

\section{Qualidade visual dos ovos (Ovoscopia)}

A qualidade visual dos ovos não apresentou problemas em nenhuma das amostras avaliadas, indicando que todas estavam dentro das condições normais e dentro dos padrões de consumo. Barbosa Filho (2004) observou que o sistema de criação em gaiolas apresentou maior quantidade de ovos sujos e quebrados, indicando assim pior desempenho desse sistema de criação sob o ponto de vista da qualidade visual externa dos ovos. Esses resultados diferiram dos obtidos, pois esse sistema não apresentou problema, concordando com os trabalhos de Abrahamsson (1996) e Appleby (1998).

Jacob et al. (2000), observaram que linhagens vermelhas, produtoras de ovos de casca marrom, em geral, apresentavam maior número de ovos com pontos de sangue, resultantes de hemorragias internas em pequenos vasos sangüíneos situados no ovário ou no oviduto das aves. Quando ocorrem na gema são geralmente ocasionados por hemorragias no ovário. Embora a linhagem utilizada seja classificada como vermelha, não foi detectado esse tipo de problema.

\section{Peso}

A análise estatística das médias dos pesos dos ovos revelou não existir diferença significativa desse parâmetro de qualidade, com relação à adição do urucum à ração dos animais. $\mathrm{O}$ peso que as amostras apresentaram ficou entre 59,61g e 59,12g, tendo como média 59,41g.

Barbosa Filho (2004) encontrou variação entre os pesos de 3,2g e 2,4g para linhagem Hy-Line brown, respectivamente para os sistema de criação em cama e para o sistema de criação em gaiola. Os ovos tiveram pesos 
Tabela 1 - Médias dos parâmetros de qualidade física: peso, gravidade específica, altura de albúmen e unidade Haugh.

\begin{tabular}{lcccc}
\hline \multicolumn{1}{c}{ Tratamento } & $\begin{array}{c}\text { Peso } \\
(\mathrm{g})\end{array}$ & $\begin{array}{c}\text { Gravidade } \\
\text { específica }\end{array}$ & $\begin{array}{c}\text { Altura do Albumen } \\
(\mathrm{mm})\end{array}$ & $\begin{array}{c}\text { Unidade } \\
\text { Haugh }\end{array}$ \\
\hline T1 (Controle) & $59,61 \pm 4,5 \mathrm{a}^{1}$ & $1,09 \pm 0,0 \mathrm{a}$ & $8,62 \pm 1,4 \mathrm{a}$ & $92,29 \pm 6,7 \mathrm{~b}$ \\
T2 (adição 0,5\% de urucum) & $59,12 \pm 3,4 \mathrm{a}$ & $1,09 \pm 0,1 \mathrm{a}$ & $9,29 \pm 1,4 \mathrm{a}$ & $95,86 \pm 7,6 \mathrm{a}$ \\
T3 (adição 1,0 \% de urucum) & $59,55 \pm 4,6 \mathrm{a}$ & $1,11 \pm 0,1 \mathrm{a}$ & $8,77 \pm 1,8 \mathrm{a}$ & $92,72 \pm 8,9 \mathrm{ab}$ \\
T4 (adição 1,5\% de urucum) & $59,53 \pm 3,7 \mathrm{a}$ & $1,10 \pm 0,0 \mathrm{a}$ & $8,77 \pm 1,3 \mathrm{a}$ & $92,99 \pm 5,9 \mathrm{ab}$ \\
T5 (adição 2,0 \% de urucum) & $59,25 \pm 4,2 \mathrm{a}$ & $1,09 \pm 0,0 \mathrm{a}$ & $8,87 \pm 1,3 \mathrm{a}$ & $93,99 \pm 6,9 \mathrm{ab}$ \\
\hline
\end{tabular}

${ }^{1}$ Médias \pm Desvio padrão. Médias seguidas da mesma letra na coluna não diferem entre si pelo teste de Tukey $(p>0,05)$.

Tabela 2 - Médias dos parâmetros de qualidade física: espessura da casca, altura da gema, diâmetro de gema e índice de gema.

\begin{tabular}{lcccc}
\hline \multicolumn{1}{c}{ Tratamento } & $\begin{array}{c}\text { Espessura da casca } \\
(\mathrm{mm})\end{array}$ & $\begin{array}{c}\text { Altura da gema } \\
(\mathrm{mm})\end{array}$ & $\begin{array}{c}\text { Diâmetro da gema } \\
(\mathrm{mm})\end{array}$ & $\begin{array}{c}\text { Índice de } \\
\text { gema }\end{array}$ \\
\hline T1 (Controle) & $0,58 \pm 0,0 \mathrm{a}^{1}$ & $16,38 \pm 0,8 \mathrm{a}$ & $38,00 \pm 0,1 \mathrm{a}$ & $0,43 \pm 0,3 \mathrm{ab}$ \\
T2 (adição 0,5\% de urucum) & $0,59 \pm 0,0 \mathrm{a}$ & $16,46 \pm 0,8 \mathrm{a}$ & $37,50 \pm 0,2 \mathrm{a}$ & $0,44 \pm 0,3 \mathrm{a}$ \\
T3 (adição 1,0 \% de urucum) & $0,59 \pm 0,0 \mathrm{a}$ & $16,46 \pm 1,1 \mathrm{a}$ & $37,90 \pm 0,1 \mathrm{a}$ & $0,43 \pm 0,3 \mathrm{ab}$ \\
T4 (adição 1,5\% de urucum) & $0,59 \pm 0,0 \mathrm{a}$ & $16,07 \pm 0,7 \mathrm{a}$ & $38,00 \pm 0,1 \mathrm{a}$ & $0,42 \pm 0,2 \mathrm{~b}$ \\
T5 (adição 2,0 \% de urucum) & $0,58 \pm 0,0 \mathrm{a}$ & $16,27 \pm 1,0 \mathrm{a}$ & $37,70 \pm 0,1 \mathrm{a}$ & $0,43 \pm 0,3 \mathrm{ab}$ \\
\hline
\end{tabular}

${ }^{1}$ Médias \pm Desvio padrão. Médias seguidas da mesma letra na coluna não diferem entre si pelo teste de Tukey $(\mathrm{p}>0,05)$.

médios entre $58 \mathrm{~g}$ para o sistema de criação em cama, e $56 \mathrm{~g}$ para o sistema de criação em gaiola, confirmando assim, os dados apresentados na Tabela 1.

\section{Gravidade específica}

A gravidade específica é uma estimativa da quantidade de casca depositada, e está relacionada com a porcentagem de casca. Quando a gravidade específica aumenta a resistência à quebra da casca também aumenta (OLSSON, 1934). Peebles \& McDaniel (2004) consideraram em seu trabalho o valor da gravidade específica 1,0800 com o valor limite entre baixa e alta qualidade da casca dos ovos. Mendonça Júnior et al. (1999) apresentaram valor inferior de gravidade específica $(1,0777)$.

Com relação à gravidade específica dos ovos, podese observar na Tabela 1, que as amostras não diferiram entre si $(\mathrm{p}>0,05)$ das médias dos valores avaliados, com relação aos ovos tratados, demonstrando que o urucum não interfere negativamente na densidade dos ovos. Segundo Silva (2004), a densidade dos ovos não pode ser inferior a 1,080. Todos os tratamentos apresentaram valores superiores, indicando que os ovos produzidos foram de boa qualidade considerando o parâmetro densidade.

A gravidade específica é importante para o produtor de ovos, pois está diretamente ligada à qualidade da casca dos ovos, sendo um método rápido e fácil, não-destrutivo e que fornece melhor caracterização da qualidade (BARBOSA FILHO, 2004; HAMILTON, 1982; SECHINATO, 2003). Alterações na medida da gravidade específica podem ocorrer devido à concentração das soluções quando ocorrem perdas por evaporação, e também por fissuras na casca ou movimentação na água (FREITAS et al., 2004).

\section{Unidade Haugh}

A altura do albúmen é utilizada para relacionar com o peso dos ovos na unidade Haugh e o diâmetro do albúmen na verificação do índice de albúmen. Entre os tratamentos não houve diferença significativa $(\mathrm{p}>0,05)$ para esse quesito, demonstrando que não houve influência da adição de urucum na ração, conforme observa-se na Tabela 1.

O parâmetro de qualidade Unidade Haugh apresentou diferença estatística significativa, com relação ao tratamento que recebeu $0,5 \%$ de urucum na ração e o controle $(\mathrm{p} \leq 0,05)$.

Barbosa Filho (2004) observou que há influência nesse parâmetro quanto ao sistema de criação, houve queda dos valores da unidade Haugh para os ovos postos no sistema de criação em gaiolas. 
É interessante observar que, mesmo com a adição de urucum os ovos foram classificados como do tipo AA, (unidade Haugh entre 100 a 72) (SILVA, 2004). Os ovos de qualidade AA são os melhores para cocção, possuem a casca limpa, intacta e praticamente normal; câmara-de-ar com $0,3 \mathrm{~cm}$ ou menos de profundidade em formato regular; clara límpida e firme; gema bem centralizada, contorno ligeiramente definido e sem defeitos. Quando quebrados em superfície plana, esses ovos devem se apresentar cobrindo uma pequena área; com clara abundante e a clara espessa cercando a gema; há pequena quantidade de clara fina; gema redonda e proeminente (GRISWOLD, 1972). Mendonça Júnior et al. (1999) apresentaram valor de 86,7 para unidade Haugh, estando os ovos classificados em tipo AA.

Não houve diferença com a utilização do urucum na ração quanto à classificação dos ovos. Considerando que as aves estavam em início de postura, era esperado que os ovos recém- coletados apresentassem excelentes valores, segundo Griswold (1972) e Souza et al. (1994).

\section{Espessura da Casca}

A adição de urucum na ração das poedeiras não teve influência significativa $(p>0,05)$ sobre a espessura das cascas das amostras analisadas. O mesmo ocorreu com a altura do albúmen, peso, unidade Haugh e gravidade específica.

Os valores da espessura da casca foram maiores do que o encontrado por Mendonça Júnior et al. (1999), que obtiveram valor médio de 0,362 mm. Barbosa Filho (2004), encontrou valores médios de $0,410 \mathrm{~mm}$ para animais da raça Hy-Line Brown no sistema de criação em gaiolas. Emery et al. (1984), confirmado por Barbosa Filho (2004) e Mahmoud (1996), verificaram que há uma queda acentuada na espessura da casca quando o animal passa por estresse térmico, decorrente da diminuição do balanço de cálcio no sangue. Esse balanço é afetado quando a ave se encontra em condições de alta temperatura, diminuindo a quantidade de cálcio no plasma e comprometendo a formação da casca do ovo. Barbosa Filho (2004) também observou que, quanto às condições de criação, de modo geral, o sistema de criação em gaiolas foi o que apresentou maiores quedas nos valores médios da espessura da casca, tanto em condições normais, quanto de estresse, porém em condições normais (sem estresse térmico), a linhagem Hy-Line W36 (branca) apresentou valores superiores de espessura de casca (média de $0,460 \mathrm{~mm}$ ), diferindo dos valores encontrados (Tabela 2), onde os valores médios ficaram em torno de $0,580 \mathrm{~mm}$.

O parâmetro espessura da casca também é de grande interesse para os produtores de ovos, uma vez que problemas como perda de ovos por quebra ou rachaduras poderão trazer prejuízos, além de indicar também que, provavelmente, a causa do problema esteja ocorrendo devido à falhas de ambiência dentro das instalações onde as aves se encontram (BARBOSA FILHO, 2004). Pelos resultados encontrados na Tabela 2, observa-se que não há interferência do urucum nesse parâmetro. Segundo Jacob et al. (2000), problemas na casca podem resultar em baixa classificação dos ovos, o que poderá causar desvalorização do produto no mercado.

\section{Índice de Gema}

Não houve diferença significativa $(p>0,05)$ entre os tratamentos para o índice de gema, indicando que a adição de urucum não exerceu influência, conforme observa-se na Tabela 2. O índice de gema está dentro da normalidade $(0,3$ a 0,5$)$ e também dentro dos valores encontrados por Barbosa Filho (2004), que encontrou valores de 0,44 a 0,41, para a linhagem Hy-Line Brown . Barbosa Filho (2004) também observou que, quanto à linhagem Hy-Line Brown, não houve diferença para esse parâmetro de qualidade entre as condições ambientais avaliadas; no entanto, quando analisou o valor médio de índice de gema, para os sistemas de criação (cama ou gaiola), verificou que ocorreu redução significativa nos valores de índice de gema, no sistema de criação em gaiola.

\section{CONCLUSÃO}

De acordo com condições em que foi realizado o experimento pode-se concluir que: os tratamentos contendo urucum não interferiram na qualidade física dos ovos.

O uso do urucum na alimentação de poedeiras pode incentivar o cultivo do urucum, espécie nativa do nosso país, constituindo-se em alternativa de fonte de renda para quem vive da agricultura.

\section{AGRADECIMENTOS}

Ao Dr. Camilo Flamarion Franco, diretor técnico da EMEPA, pelas amostras de urucum e ao Eng. Agrônomo Sílvio Bertolucci, pelos animais.

\section{REFERÊNCIAS BIBLIOGRÁFICAS}

ABRAHAMSSON, P. Furnished cages and aviaries for laying hens: effects on production, health and use of facilities. Swedish: Swedish University of Agricultural Science, 1996. (Report, 234).

APPLEBY, M. C. Modification of laying hen cages to improve behaviour. Poultry Science, Ithaca, v. 77, p. 18281832, 1998. 
ARAYA, H. H.; MURILLO, M. R.; VARGAS, E. G.; DELGADO, J. M. Composición y empleo del achiote (Bixa orellana L.) en raciones para gallinas ponedoras, para la pigmentacion de la yema del huevo. Agronomia Costarricense, San Jose, v. 1, n. 2, p. 143-150, 1977.

BARBOSA FILHO, J. A. D. Avaliação do bem-estar de aves poedeiras em diferentes sistemas de produção e condições ambientais, utilizando análise de imagem. 2004. $123 \mathrm{f}$. Dissertação (Mestrado em Agronomia) - Escola Superior de Agricultura “Luiz de Queiroz”, Piracicaba, 2004.

BATISTA, C. L. L. C.Produção e avaliação da estabilidade de corante hidrossolúvel de urucum. Viçosa: UFV, 1994. 71 p.

BOARD, R. G.; LOCK, J.; DOLMAN, J. The egg: a compartmentalized, asseptically package food. In: BOARD, R. G.; FULLER, R. Microbiology of the avian egg. London: Chapman \& Hall, 1994. p. 95-99.

CATÁLOGO RURAL. Disponível em: <http:// 'www.agrov.com/_egetais/frutas/index.htm?. Acesso em: 30 nov. 2005 .

EMERY, D. A.; VOHRA, P.; ERNEST, R. A.; MORRISON, S. $\mathrm{R}$. The effect of cyclic and constant ambient temperatures on feed consumption, egg production, egg weight, and shell thickness of hens. Poultry Science, Ithaca, v. 63, p. 2027-2035, 1984.

ENGLERT, S. I. Avicultura: tudo sobre raças, manejo e alimentação. 7. ed. atual. Guaíba: Livraria e Editora Agropecuária, 1998. 238 p.

FAO. Necesidades de vitamina $\mathrm{A}$, hierro, folato y vitamina $\mathbf{B}_{12}$ : informe de uma consulta mista FAO/OMS de expertos. Roma, 1991. 121 p. (Serie Estúdios FAO Alimentación y Nutricición).

FRANCO, C. F. O.; SILVA, F. C. P.; CAZÉ FILHO, J.; BARREIRO NETO, M.; SÃO JOSÉ, A. R.; REBOUÇAS, T. N. H.; FONTINÉLLI, I. S. C. Urucuzeiro: agronegócio de corantes naturais. João Pessoa: Emepa, 2002. 120 p.

FREITAS, E. R.; SAKOMURA, N. K.; GONZALEZ, M. M.; BARBOSA, N. A. A. Comparação de métodos de determinação da gravidade específica da gravidade específica de ovos de poedeiras comerciais. Pesquisa Agropecúaria Brasileira, Brasília, v. 39, n. 5, p. 509-512, 2004.

GRISWOLD, R. M. Estudo experimental dos alimentos. São Paulo: USP, 1972. 469 p.
HAMILTON, R. M. G. Methods and factors that affect the measurement of egg shell quality. Poultry Science, Ithaca, v. 61, p. 2022-2039, 1982.

JACOB, J. P.; MILES, R. D.; MATHER, F. B. Egg quality. Florida: Institute of Food and Agricultural ScienceUniversity of Florida, 2000. 11 p. (Bulletin, PS24).

JOLY, A. B. Botânica: introdução à taxonomia vegetal. São Paulo: Nacional, 1993. 777 p.

MAHMOUD, K. Z.; BECK, M. M.; SCHEIDELER, S. E.; FORMAN, M. F.; ANDERSON, K. P.; KACHMAN, S. D. Acute high environmental temperature and calciumestrogen relationships in the hens. Poultry Science, Ithaca, v. 75, p. 1555-1562, 1996.

MENDONÇA JÚNIOR, C. X.; WATANABE, C.; MORI, A. V.; SANTOS, C. O. F.; ALMEIDA, C. R. M. Efeitos de níveis de cobre suplementar na dieta sobre o desempenho produtivo, colesterol na gema e lípides no plasma sangüíneo de poedeiras comerciais. Brazilian Journal of Veterinary Research and Animal Science, São Paulo, v. 36, n. 6, 1999.

OLIVEIRA, B. L.; VALLE, R. H. P.; BRESSAN, M. C.; CARVALHO, E. P. Tecnologia de ovos. Lavras: UFLA/ FAEPE, 2001. $75 \mathrm{p}$.

OLSSON, N. Studies on specific gravity of hen's eggs. Otto Harrassolvitz: Leipzig, 1934. 89 p.

PEEBLES, E. D.; McDANIEL, C. D. A practical manual for understanding the shell structure of broiler hatching eggs and measurements of their quality. Mississipi: State University, 2004. 16 p. (Bulletin, 1139).

SECHINATO, A. S. Efeito da suplementação dietética com micro minerais orgânicos na produção e qualidade de ovos de galinhas poedeiras. 2003. 59 p. Dissertação (Mestrado em Medicina Veterinária) - Universidade de São Paulo, São Paulo, 2003.

SILVA, F. H. A. Curso teórico-prático sobre técnicas básicas de avaliação de qualidade do ovo. Piracicaba: ESALQ, 2004.

SOUZA, H. B. A.; SOUZA, P. A.; BROGNONI, E.; ROCHA, O. E. Influência da idade sobre a qualidade dos ovos. Cientifica, São Paulo, v. 22, n. 2, p. 217-226, 1994. 